\title{
Dynamics of Classical Wave Scattering by Small Obstacles
}

\author{
Gerrit E. W. Bauer, ${ }^{1}$ Mauro S. Ferreira, ${ }^{1}$ and Cees P. A. Wapenaar ${ }^{2}$ \\ ${ }^{1}$ Theoretical Physics Group, Department of Applied Physics and Delft Institute of Microelectronics and Submicron Technology, \\ Delft University of Technology, Lorentzweg 1, 2628 CJ Delft, The Netherlands \\ ${ }^{2}$ Department of Applied Earth Sciences, Delft University of Technology, Mijnbouwstraat 128, 2628 RX Delft, The Netherlands
}

(Received 16 May 2001; published 27 August 2001)

\begin{abstract}
A causality problem in the time-dependent scattering of classical waves from point scatterers is pointed out and analyzed. Based on an alternative model, the leading pole approximation of the exact scattering matrix of the square-well potential, transparent expressions for the time- and position-dependent Green function in a disordered medium are derived.
\end{abstract}

DOI: $10.1103 /$ PhysRevLett.87.113902

Wave propagation in complex media is a large and interdisciplinary field of research with many unsolved problems that are scientifically challenging and technologically important [1]. Electromagnetic and acoustic waves are massless and described by a "classical" wave equation which is second order in the time derivative, whereas "quantum" particle waves are governed by the Schrödinger equation which is first order in $\partial / \partial t$. Scalar classical waves at a fixed frequency $\omega$ are thus equivalent to a particle wave at energy $E \sim \omega^{2}$. The analogies between the classical and quantum problems indeed lead to many cross fertilizations since solutions obtained in one field can be carried over to the other. For example, the discovery of localization of classical waves in random media [2] was stimulated by earlier work on electron localization [3]. Disordered systems have to be treated by statistical methods, for example, by an ensemble average over the configurations of randomly distributed model scatterers. The (diagrammatic) perturbation theory for potential disorder in metals [4] has been helpful in understanding classical wave propagation, including (weak) wave localization [5]. An essential ingredient in these calculations is a mathematically simple yet physically meaningful and well-behaved model for the disorder. The point ( $\delta$-function) scatterer is often the basic building block for classical and quantum problems, because of its simple scattering amplitude. The associated ultraviolet divergence must be regularized, however $[5,6]$.

Most previous studies of classical waves in complex systems have been limited to monochromatic, steady-state wave fields, but the propagation of short pulses containing a broad band of frequencies is of considerable interest as well. Concrete challenges are provided by seismic waves excited by earthquakes or artificial explosions in terms of first arrival times [7] and the waves which trail behind (the so-called "coda") [8]. The power spectrum of the coda has received theoretical attention [9], also in the framework of random matrix theory [10]. Random matrix theory of transport, until now mainly applied to quantum problems [11], identifies the "geometrical" or "universal" features of an observable that do not depend on the microscopic details of the system. Alternatively, however, one may pursue a microscopic approach trying to answer, for instance, what
PACS numbers: 42.25.Dd, 91.30.Rz

sort of information about the geophysical complexity of the subsurface can be distilled from the total seismic trace. To this end, models and approximations must be found which are well-behaved and sufficiently accurate over the whole complex frequency plane. In this Letter, we point out that point defects interacting with classical waves are responsible for wrong analytical properties of the scattering matrix in the frequency domain equivalent to noncausality in the time domain, which appears to have escaped attention in the literature. We propose a different model that has all the practical advantages of point scatterers but gives a causal response with the additional bonus that an ultraviolet cutoff is not needed. For simplicity, we limit attention in the following to scalar fields referring to [6] for a discussion of point scatterers for vector fields. After treating the simple one-dimensional (1D) problem, we turn to the practically more interesting three-dimensional (3D) space. The usefulness of the new model is illustrated by a calculation of the amplitude coda in a random medium with a low density of weak scatterers.

Let us consider a classical scalar field in 1D with spatially varying celerity (wave velocity) $c(x)$. The $1 \mathrm{D}$ problem corresponds to a layered system with planar sources and without lateral disorder, which is relevant as an approximation for, e.g., the seismics of rock sediments [12]. The wave potential $\phi_{i}(x ; \omega)$ at a given frequency $\omega$ is then governed by the eigenvalue equation:

$$
\left\{-\frac{\partial^{2}}{\partial x^{2}}+V(x, \omega)\right\} \phi_{i}(x ; \omega)=E_{i}(\omega) \phi_{i}(x ; \omega),
$$

introducing the frequency dependent "scattering potential",

$$
V(x, \omega)=\frac{\omega^{2}}{c_{0}^{2}}\left(1-\frac{c_{0}^{2}}{c(x)^{2}}\right),
$$

which is "attractive" $(V<0)$ when the local celerity $c(x)$ is smaller than the reference celerity $c_{0}$ (as for air bubbles in water) and "repulsive" $(V>0)$ when $c(x)>c_{0}$ (as for liquid mercury in water). The physical quantity of interest is the retarded Green function or point source propagator ( $\eta$ is a positive infinitesimal): 


$$
G\left(x, x^{\prime} ; \omega\right)=\sum_{i} \frac{\phi_{i}(x ; \omega) \phi_{i}\left(x^{\prime} ; \omega\right)^{*}}{\frac{\omega^{2}}{c_{0}^{2}}-E_{i}(\omega)+i \eta \operatorname{sgn}(\omega)},
$$

which is an observable for classical fields. A useful concept is the scattering matrix which connects the amplitude of incoming and outgoing amplitudes of a given scattering region [13]. In $1 \mathrm{D}$, the scattering matrix has rank 2 and eigenvalues $S_{\ell}$, for the even channel $\ell=s$ and the odd channel $\ell=a$.

Let us consider a point model scattering potential $V_{\delta}(x, \omega) \approx \gamma\left(\omega / c_{0}\right)^{2} \delta(x)$ with a scattering strength parametrized by $\gamma$. A straightforward calculation gives

$$
S_{s}^{\delta}(\omega)=-\frac{\omega+i \Gamma_{\delta}}{\omega-i \Gamma_{\delta}} ; \quad S_{a}^{\delta}=1,
$$

where $\Gamma_{\delta}=2 c_{0} / \gamma$. The reflected amplitude from a pulse at time $t^{\prime}=0$ and $x^{\prime}=L$ observed at time $t$ and the same position $x=L$ can be obtained by contour integration, collecting the pole at $\omega=i \Gamma_{\delta}$ :

$$
\begin{gathered}
G(L, L ; t)=\int \frac{d \omega}{2 \pi} \frac{c}{2 i \omega} \frac{1}{2}\left(S_{s}-S_{a}\right) e^{i \omega 2 L / c_{0}} e^{-i \omega t}, \\
\left.=\begin{array}{c}
\frac{c_{0}}{2} e^{\Gamma_{\delta}\left(t-2 L / c_{0}\right)} \Theta\left(t-\frac{2 L}{c_{0}}\right) \\
-\frac{c_{0}}{2} e^{\Gamma_{\delta}\left(t-2 L / c_{0}\right)} \Theta\left(\frac{2 L}{c_{0}}-t\right)
\end{array}\right\} \text { for }\left\{\begin{array}{l}
\Gamma_{\delta}<0 \\
\Gamma_{\delta}>0
\end{array},\right.
\end{gathered}
$$

where $\Theta$ is the step function. Clearly, the result is well behaved when the scattering potential is attractive $\left(\Gamma_{\delta}<\right.$ 0 ), but violates causality for a repulsive scatterer, since the reaction appears before the action.

In order to shed light on this artifact, let us consider a finite square-well insertion of thickness $d$ and celerity $c$ centered at the origin and embedded into the infinite medium with celerity $c_{0}$ :

$$
\begin{aligned}
V(x, \omega)= & \frac{\omega^{2} d}{c_{0}^{2}}\left(1-\frac{c_{0}^{2}}{c^{2}}\right) \\
& \times \frac{\Theta(d / 2-x)-\Theta(x-d / 2)}{d} .
\end{aligned}
$$

In the limit of vanishing $d$, the last factor approaches the $\delta$ function. For a finite scattering amplitude in this limit $\mid 1-$ $c_{0}^{2} / c^{2} \mid$ must scale similar to $d^{-1}$, which is possible when $c \ll c_{0}$. When $c>c_{0}$, the scattering strength necessarily vanishes when $d \rightarrow 0$. A repulsive point scatterer can be realized only by an imaginary celerity in the insertion, which causes the noncausality.

Obviously, a better behaved model which retains the attractive features of the point scatterer is necessary. We show now that a single pole approximation of the scattering matrix [13] satisfies these requirements. The eigenvalues of the $S$ matrix for the potential (7) reads

$$
S_{s / a}= \pm e^{-i \omega d / c_{0}} \frac{\mathfrak{r}_{0}\left(1-e^{2 i \omega d / c}\right) \mp\left(1-\mathfrak{r}_{0}^{2}\right) e^{2 i \omega d / c}}{1-\mathfrak{r}_{0}^{2} e^{2 i \omega d / c}},
$$

where $\mathfrak{r}_{0}=\left(c-c_{0}\right) /\left(c+c_{0}\right)$ is the reflection coefficient of an isolated celerity discontinuity [14]. The denominators of both $S_{s / a}$ vanish at

$$
\omega_{n}=n \frac{\pi c}{d}+i \frac{c}{d} \log \left|\mathfrak{x}_{0}\right|=n \Omega_{0}+i \Gamma .
$$

All resonances (or quasinormal modes [15]) are equally broadened by $\Gamma$ and define the product representation of the scattering matrix (with the proper global phase factor) [16]:

$$
\begin{aligned}
S_{s / a}(\omega)= & -e^{-i \omega d / c_{0}} \frac{\omega+i \Gamma}{\omega-i \Gamma} \\
& \times \prod_{n=1}^{\infty} \frac{(\omega+i \Gamma)^{2}-\left(n \Omega_{0}\right)^{2}}{(\omega-i \Gamma)^{2}-\left(n \Omega_{0}\right)^{2}} .
\end{aligned}
$$

For the attractive (repulsive) scatterer the residues vanish when $n$ is odd (even) for $\ell=s$ and $n$ is even (odd) for $\ell=a$. The poles are always in the lower half of the complex frequency plane as required by causality.

We can now introduce an approximation in which only the purely imaginary pole is taken into account, which is formally justified for long time scales $\Delta t=t-2 L / c_{0} \gg$ $d / c$ and high reflectivities $1-\mathfrak{r}_{0}^{2} \ll 1$. For an attractive scatterer,

$$
S_{s}^{-} \approx-e^{-i \omega d / c_{0}} \frac{\omega+i \Gamma}{\omega-i \Gamma} ; \quad S_{a}^{-} \approx 1,
$$

whereas for the repulsive scatterer the roles of the odd and even scattering channels are reversed. The time-dependent reflection amplitude is now well behaved:

$$
G^{ \pm}(L, L ; t)=\mp \Theta\left(t-\frac{2 L}{c_{0}}\right)\left|\mathfrak{r}_{0}\right|^{c / d\left[t-\left(2 L / c_{0}\right)\right]+c / c_{0}} .
$$

Let us now turn to the $3 \mathrm{D}$ problem. The $s$-wave scattering matrix of a point scatterer at the origin $V(\vec{r}, \omega)=$ $\gamma\left(\omega / c_{0}\right)^{2} \delta(\vec{r})$ reads [5]

$$
S_{0}^{\delta}(\omega)=-\frac{\omega+i \frac{4 \pi c}{\gamma}\left(\frac{c_{0}^{2}}{\omega^{2}}-\frac{1}{k^{* 2}}\right)}{\omega-i \frac{4 \pi c}{\gamma}\left(\frac{c_{0}^{2}}{\omega^{2}}-\frac{1}{k^{* 2}}\right)},
$$

where $\left(k^{*}\right)^{-1}=\sqrt{-\gamma k_{c}}$ and $k_{c}$ is a high momentum cutoff which is necessary to regularize the point scatterer model. Clearly, $S_{0}^{\delta}$ is unitary for attractive scatterers only $(\gamma<0)$ and does not have the correct analytical properties for all $\gamma$. This does not disqualify point scatterers for studies of low celerity insertions and monochromatic illumination, but they are clearly unsuitable for pulsed, broadband sources. We can find a remedy along the lines sketched above for 1D using a spherical square-well scatterer with diameter $d$ and velocity $c$. The higher-angular momentum channels are of higher order in $\omega d / c_{0}$ and can therefore be disregarded when $\Delta t \gg d / c_{0}$. The $s$-channel eigenvalue of the $S$ matrix reads [13]

$$
S_{0}(\omega)=-e^{-i \omega d / c_{0}}\left(1+\frac{2 i}{\frac{c_{0}}{c} \cot \frac{\omega d}{2 c_{0}}-i}\right) .
$$


For repulsive scatterers, its poles are at $\omega_{0, n}^{+}=2 n \pi c / d+$ $i \Gamma$ with $\Gamma=\frac{c}{d} \log \left|\mathfrak{x}_{0}\right|$. Taking into account only the lowest, evanescent mode,

$$
S_{0}^{+}(\omega) \approx-e^{-i \omega d / c_{0}} \frac{\omega+i \Gamma}{\omega-i \Gamma}
$$

For attractive scatterers, there is no purely imaginary pole since $\omega_{0, n}^{-}=(2 n+1) \pi c / d+i \Gamma$ and the lowest approximation involves two poles:

$$
S_{0}^{-}(\omega) \approx e^{-i \omega d / c_{0}} \frac{(\omega+i \Gamma)^{2}+\left(\frac{\pi c}{d}\right)^{2}}{(\omega-i \Gamma)^{2}+\left(\frac{\pi c}{d}\right)^{2}} .
$$

The similarities and differences between 1D and 3D are notable. The exact propagator in time,

$$
\Delta G_{\ell=0}(L, L ; t)=-\frac{c_{0}}{8 \pi L^{2}}\left[\mathfrak{r}_{0} \Theta\left(t-\frac{2 L-d}{c_{0}}\right)-\Theta\left(t-\frac{2 L}{c_{0}}\right) \frac{4 c_{0} c}{\left(c+c_{0}\right)^{2}} \sum_{n=0}^{\infty} \Theta\left(t-\frac{2 L-d}{c_{0}}-\frac{(n+1) d}{c}\right)\right],
$$

is then approximated for $c>c_{0}$ as

$$
\begin{aligned}
\Delta G_{\ell=0}^{+}(L, L ; t) & \approx-\frac{c_{0}}{8 \pi L^{2}}\left[\Theta\left(t-\frac{2 L}{c_{0}}\right)+\Theta\left(t-\frac{2 L-d}{c_{0}}\right)\left(2 \mathfrak{x}_{0}^{(c / d)\left\{t-\left[(2 L-d) / c_{0}\right]\right\}}-1\right)\right] \\
& \stackrel{d \rightarrow 0}{\approx}-\frac{c_{0}}{4 \pi L^{2}} \Theta\left(t-\frac{2 L}{c_{0}}\right) \mathfrak{r}_{0}^{(c / d)\left[t-(2 L) / c_{0}\right]},
\end{aligned}
$$

and $c<c_{0}$ as

$$
\begin{aligned}
& \Delta G_{\ell=0}^{-}(L, L ; t) \approx-\frac{c_{0}}{8 \pi L^{2}} \Theta\left(t-\frac{2 L}{c_{0}}\right)+\frac{c_{0}}{8 \pi L^{2}} \Theta\left(t-\frac{2 L-d}{c_{0}}\right) \\
& \times\left\{1+\frac{4 \log \left|\mathfrak{r}_{0}\right|}{\pi}\left|\mathfrak{r}_{0}\right|^{(c / d)\left[t-(2 L-d) / c_{0}\right]} \sin \left[\frac{\pi c}{d}\left(t-\frac{2 L-d}{c_{0}}\right)\right]\right\} \\
& \stackrel{d \rightarrow 0}{\approx} \frac{c_{0}}{2 \pi L^{2}} \Theta\left(t-\frac{2 L}{c_{0}}\right) \frac{\log \left|\mathfrak{r}_{0}\right|}{\pi}\left|\mathfrak{r}_{0}\right|^{(c / d)\left[t-(2 L-d) / c_{0}\right]} \sin \left[\frac{\pi c}{d}\left(t-\frac{2 L}{c_{0}}\right)\right] .
\end{aligned}
$$

In Eqs. (19) and (21), the exponential phase factors in Eqs. (15) and (16) have been approximated by unity. In Figure 1 we compare the exact reflection amplitudes (17) with the two approximations. The envelope of the response is quantitatively well represented by the lowest pole approximation. The subsequent neglect of the phase shift is a rather crude approximation for a single scatterer, but should be allowed in a many-scatterer configuration to be considered next.

Let us now turn to the response of a disordered medium. The approximate scattering matrices derived above are directly related to the t-matrix eigenvalues $t_{\ell}=c_{0}\left(S_{\ell}-\right.$ $1) / 2 i \omega$, which are proper partial sums in a perturbation theory of disordered systems with nonoverlapping scatterers [5]. In the limit of a low density $n$ of randomly distributed scatterers the interference between waves multiply scattered by different sites may be disregarded. When all scatterers are the same, the ensemble averaged Green function in wave vector $\vec{q}$ and frequency space reads (in 3D)

$$
\langle G(q ; \omega)\rangle^{-1}=\left(\frac{\omega}{c_{0}}\right)^{2}-q^{2}-n \mathrm{t}_{0},
$$

For repulsive scatterers we employ the approximation (19). Transformation into time and position space gives

$$
\begin{aligned}
\langle G(r ; t)\rangle= & -\frac{\Theta\left(t-r / c_{0}\right)}{2 \pi r} \int_{0}^{\infty} \frac{d \omega}{2 \pi} \cos (\omega t-|\operatorname{Re} \kappa| r) \\
& \times e^{-|\operatorname{Im} \kappa| r},
\end{aligned}
$$

where $\kappa=\sqrt{\left(\omega / c_{0}\right)^{2}-n \mathrm{t}_{0}}$ is the "renormalized" wave vector. In the weak scattering limit, which holds for time scales $\Delta t \gg|\Gamma|^{-1}$

$$
\begin{aligned}
\langle G(r ; t)\rangle= & -\frac{e^{-\left(2 \pi n c_{0}^{3} / \Gamma^{2}\right) t}}{4 \pi r} \\
& \times\left[\delta\left(t-r / c_{0}\right)+\Theta\left(t-r / c_{0}\right) C(r ; t)\right],
\end{aligned}
$$

where the delayed signal or amplitude coda reads

$$
\begin{aligned}
C(r ; t)=\frac{1}{\pi t} \int_{0}^{\infty} d x[ & \cos \left(x-\frac{r}{c_{0} t} \sqrt{x^{2}-\frac{4 \pi n c_{0}^{3} t^{2}}{\Gamma}}\right) \\
& \left.-\cos \left(x-\frac{r}{c_{0} t}\right)\right] .
\end{aligned}
$$

This integral can be solved analytically for the first arrival:

$$
\begin{aligned}
C\left(c_{0} t ; t\right)=\frac{1}{2 \pi t}[ & \sin \sqrt{\frac{4 \pi n c_{0}^{3}}{|\Gamma|}} t \\
& -\sqrt{\frac{4 \pi n c_{0}^{3}}{|\Gamma|}} t \cos \sqrt{\frac{4 \pi n c_{0}^{3}}{|\Gamma|}} t \\
& \left.-\frac{4 \pi n c_{0}^{3}}{|\Gamma|} t^{2} \operatorname{Si} \sqrt{\frac{4 \pi n c_{0}^{3}}{|\Gamma|}} t\right] .
\end{aligned}
$$

The amplitude is (Lambert-Behr) exponentially damped by the imaginary part of the t-matrix. The time-delayed signal plotted in Fig. 2 originates from multiple scattering effects on the real part of the refractive index which 

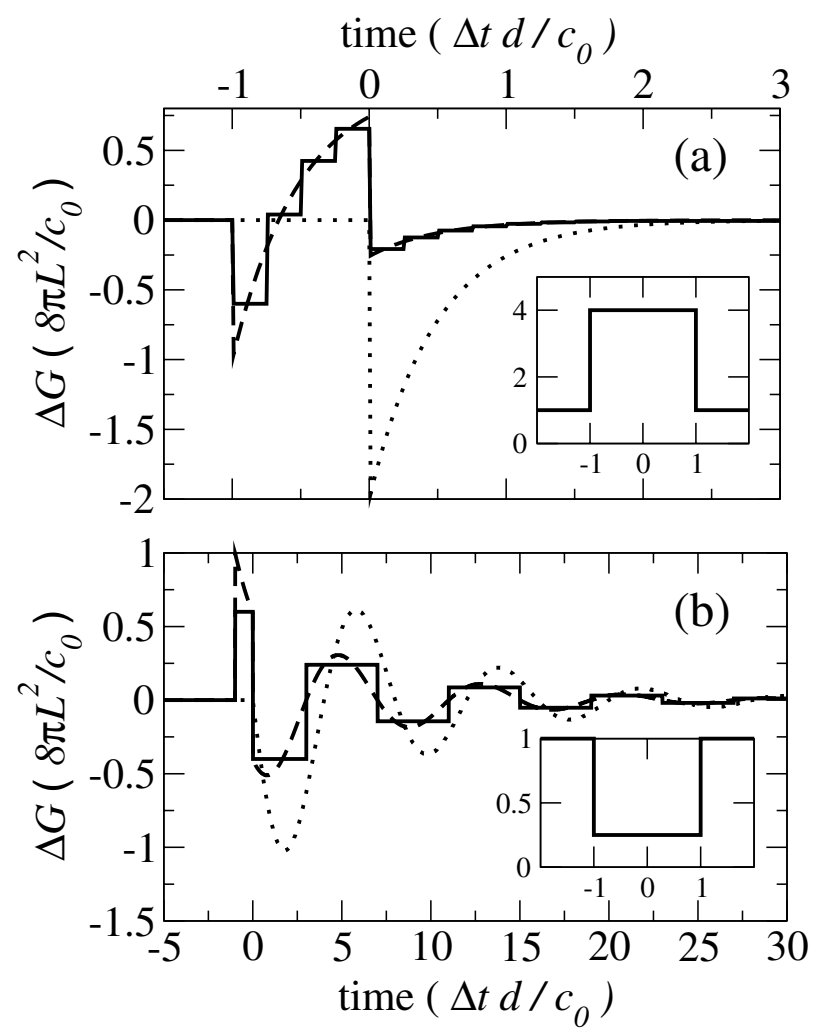

FIG. 1. Time dependence of the amplitude originating from a pulsed source and reflected by a spherical square-well potential in the $s$ channel: (a) high velocity and (b) low velocity scatterer of diameter $d . \Delta t=0$ corresponds to the nominal arrival time. The insets show the velocity profile in units of $c_{0}$ and the radius of the scatterer. Full curves denote the exact results, dashed curves correspond to the lowest pole approximation, and in the dotted curves the phase shift has been disregarded.

is equivalent with a reduced effective celerity. It depends characteristically on the microscopic parameters of the systems, viz. the density, size, and the celerity (contrast) of the scatterers. Note that the long-lived incoherent fluctuations which arrive at the detector after diffusion (the intensity coda) contribute only to the intensity $\left\langle G^{2}(r ; t)\right\rangle$, which is not discussed here.

In conclusion, we have revealed the inappropriateness of point scatterers for time-dependent classical wave scattering. An alternative model in terms of a lowest-order pole expansion is simple and well behaved. The propagator of a homogeneously disordered medium is calculated with the alternative model. A delayed signal or coda is found, which depends on the microscopic parameters of the scatterers. These results support speculations that the coda contains important information about a disordered medium that might be relevant for imaging applications.

We thank Alec Maassen van den Brink and Yaroslav Blanter for critically reading the manuscript and Yuli Nazarov for help in finding Eq. (26). This work is part of the research program of the "Stichting Technische Wetenschappen" (STW) and the "Stichting Fundamenteel

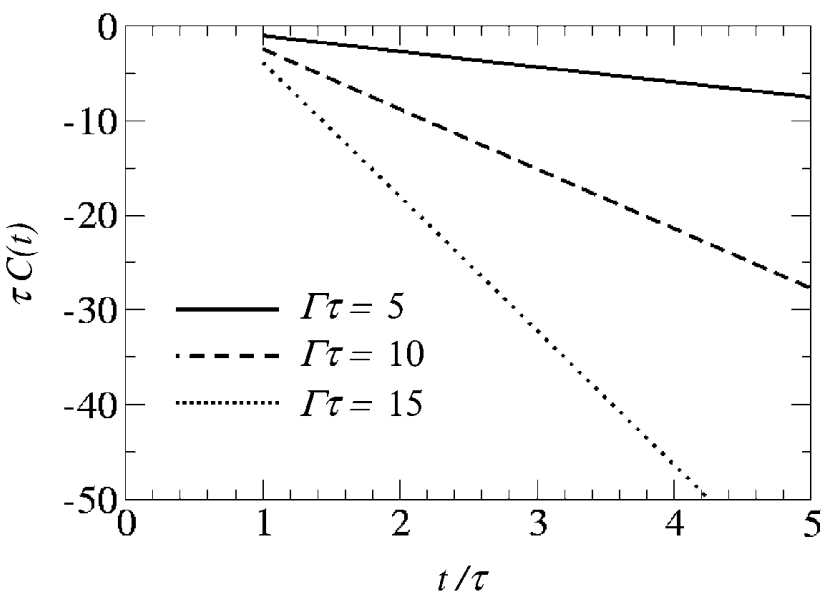

FIG. 2. Time-delayed signal in the coherently propagated amplitude $C(r=l, t)$ according to Eqs. (23) and (24) in a disordered medium with different scattering parameters $\Gamma \tau=5$ (full curve), 10 (dashed line), 15 (dotted line), where $l=c_{0} \tau=$ $\Gamma^{2} /\left(4 \pi n c_{0}^{2}\right)$ is the mean free path, $n$ is the density of scatterers, and $\Gamma$ is the imaginary part of the single-scatterer resonance frequency.

Onderzoek der Materie (FOM)". G. B. acknowledges support by the NEDO programs NTDP-98 and NAME.

[1] Waves and Imaging through Complex Media, edited by P. Sebbah (Kluwer, Dordrecht, 2001), and references therein.

[2] D. S. Wiersma et al., Nature (London) 390, 671 (1997); A. Tourin et al., Phys. Rev. Lett. 79, 3637 (1997).

[3] P. A. Lee and T. V. Ramakrishnan, Rev. Mod. Phys. 57, 287 (1985).

[4] A. A. Abrikosov, L. P. Gorkov, and I. E. Dzyaloszinski, Methods of Quantum Field Theory in Statistical Physics (Prentice-Hall, Englewood Cliffs, NJ, 1963).

[5] M.C. W. van Rossum and Th. M. Nieuwenhuizen, Rev. Mod. Phys. 71, 313 (1999).

[6] P. de Vries et al., Rev. Mod. Phys. 70, 447 (1998).

[7] M. Roth et al., Geophys. J. Int. 115, 552 (1993); J. Tworzydlo and C. W. J. Beenakker, Phys. Rev. Lett. 85, 674 (2000).

[8] H. Sato and M. C. Fehler, Seismic Wave Propagation and Scattering in the Heterogeneous Earth (Springer, New York, 1998).

[9] B. White et al., Phys. Rev. Lett. 59, 1918 (1987).

[10] M. Titov and C. W. J. Beenakker, Phys. Rev. Lett. 85, 3388 (2000).

[11] C. W. J. Beenakker, Rev. Mod. Phys. 69, 731 (1997).

[12] B. L. N. Kennett, Seismic Wave Propagation in Stratified Media (Cambridge University Press, Cambridge, England, 1983).

[13] E. Merzbacher, Quantum Mechanics (Wiley, New York, 1961).

[14] Note that mass density differences $\rho-\rho_{0}$ can be easily incorporated by $\mathfrak{x}_{0}=\left(\rho c-\rho_{0} c_{0}\right) /\left(\rho c+\rho_{0} c_{0}\right)$.

[15] E. S. C. Ching et al., Rev. Mod. Phys. 70, 1545 (1998).

[16] N. G. van Kampen, Phys. Rev. 91, 1267 (1953); H. M. Nussenzweig, Causality and Dispersion Relations (Academic, New York, 1972). 